\title{
Assessing the Performance of Eumelanin/Si Interface for Photovoltaic Applications
}

\author{
Aleandro Antidormi, ${ }^{, \dagger}$ Claudio Melis, ${ }^{\dagger}$ Enric Canadell, ${ }^{\ddagger}$ and Luciano Colombo ${ }^{\dagger}$ \\ $\dagger$ †ipartimento di Fisica, Università di Cagliari, Cittadella Universitaria, I-09042 \\ Monserrato (Ca), Italy \\ $\ddagger$ Institut de Ciència de Materials de Barcelona (ICMAB-CSIC), Campus de Bellaterra, \\ 08193 Bellaterra, Barcelona, Spain \\ E-mail: aleandro.antidormi@dsf.unica.it
}

\begin{abstract}
Among the possible applications of eumelanin (biolelectronics, biointerfaces, ...), photovoltaics is a promising field which could highly benefit from its intrinsic features (broadband UV-vis absorption, ...), with such a pigment employed as photo-active layer in hybrid solar cells. The envisioned application poses the need for a careful theoretical analysis on the adhesion properties of eumelanin on a substrate and the characterization of the hybrid system electronic features. In this work, we investigate a eumelanin $/ \mathrm{Si}$ interface, where Si plays the role of inorganic layer. By means of ab-initio calculations, we study the feasibility of the experimental formation process of eumelanin protomolecular structures in methanol ambient, evaluating the corresponding formation energy. Then, we explore the adhesion properties of eumelanin molecules on a silicon surface and extract the electronic structure of the resulting system. The corresponding band alignment is then used to address the overall photoconversion efficiency. Adopting the scheme of chemical disorder, which has been proved to successfully capture the variety of eumelanin protomolecules, we show that 1) the formation process of eumelanin
\end{abstract}


protomolecules from the constituting monomers is generally hindered in a solvent environment w.r.t vacuum; 2) key factors in improving the adhesion properties and band line-up of the molecules on an inorganic interface are the molecular electronic state and the planarity of their structures. Protomolecular models with a large number of non-terminated Oxygen atoms and endowed with an intrinsically planar character tend to bind more strongly to the surface. In addition, they are more likely to produce a favourable band alignment for photoconversion applications.

\section{Introduction}

Eumelanin is a biological pigment, widely present in living organisms, responsible for skin coloration, thermoregulation and, most importantly, photoprotection against exposure to UV radiation. ${ }^{1-3}$ Its intriguing properties, ${ }^{4}$ including broadband UV-vis absorption, ${ }^{5}$ intrinsic free radical character ${ }^{6}$ and efficient non-radiative energy dissipation, ${ }^{7,8}$ have recently attracted the interest of scientists from different disciplines toward the exploitation of synthetic eumelanin in several applications, from organic electronics ${ }^{2}$ to biointerfaces ${ }^{9,10}$ and hybrid materials ${ }^{11}$ to polymer stabilization. ${ }^{12}$

A field which could highly benefit from the employment of eumelanin is photovoltaics, with such a pigment used as photo-active layer in hybrid solar cells, ${ }^{13}$ where the light absorption process is likely to generate an exciton which dissociates at the organic/inorganic interface. ${ }^{14}$ The electron (hole) is then separately collected at the electron (hole)-accepting electrode, respectively. ${ }^{15}$ The figure in the abstract conceptualizes the operating mechanism of a hybrid solar cell together with the energy diagram of the interface between the molecular (donor) and the inorganic (acceptor) layer, corresponding to the optimal line-up for efficient photoconversion.

The driving force for the dissociation of the excitons is represented by the energy difference between the lowest unoccupied molecular orbital (LUMO) of the donor layer and the top valence band of the acceptor. Specifically, higher power conversion efficiencies are found 
when such difference is large. Moreover, in order to favour the following process of charge diffusion, the donor LUMO energy must be higher than the acceptor conduction band, while the donor highest occupied molecular orbital (HOMO) should be higher than the top valence energy. The figure in the abstract shows an example of band alignment satisfying the mentioned criteria, hence hypotetically guaranteeing good photovoltaic performance.

However, several other factors affect the overall power conversion efficiency of a hybrid solar cell, with the capability of the photo-active layer of absorbing photons ${ }^{15}$ standing high in the list. In this respect, thanks to its broad absorption spectrum, eumelanin represents a promising candidate, which could help reaching and possibly overcoming the $10 \%$ efficiency recently obtained in present hybrid solar cells. ${ }^{14}$

This scenario has engendered a growing need of asssessing the performance of eumelaninbased solar cells, which in turn translates into the requirement of a careful theoretical analysis (i) providing insights on its adhesion properties and (ii) characterising the hybrid system electronic features.

To this aim, we investigate a eumelanin/Si interface, where Silicon plays the role of inorganic layer. ${ }^{16,17}$ Our choice of Si as inorganic material is dictated by the ongoing quest for lowcost materials to be employed in photovoltaic applications. Solar cells based on crystalline silicon are known to offer high efficiency and dispose of an extremely reliable integration technology. However, they are expensive due to high temperatures required in their fabrication. ${ }^{18,19}$ A promising approach to reduce the overall production cost of new-generation devices consists in the integration of polymeric organic semiconductors into silicon-based electronic devices. This possibility potentially allows to exploit the renowned electrical and optical features of silicon together with the structural versatility and processing/mechanical properties of polymeric materials. ${ }^{20}$

In this work, we specifically investigate the formation process of eumelanin protomolecular structures in methanol ambient (methanol is the solvent most frequently employed to synthetise eumelanin) as indeed experienced experimentally. We, as well, evaluate the adhe- 
sion properties of eumelanin molecules on a $\mathrm{Si}$ [100] surface and we determine the electronic structure of the resulting Eumelanin/Silicon system and the corresponding bands alignment. The knowledge of the energy spectrum of the complete system will allow the evaluation of the hybrid cell performance. ${ }^{15}$

A major factor hindering a straightforward study of the properties outlined above is represented by the fact that the actual structure of the eumelanin molecule is mostly undefined; ${ }^{21-23}$ its determination has indeed encountered considerable difficulties primarily due to the pigment amorphous character and insolubility in all solvents. Several plausible protomolecular structures have been proposed in the recent literature with the objective of explaining the broad optical absorption spectrum in biological systems. ${ }^{24,25}$ Based on the investigation efforts made to-date, there is a general agreement in that all the synthetic eumelanin protomolecules derive from the 5,6-dihydroxy-indole (DHI) monomer and its redox forms (see figure 1); these basic components are covalently bonded to form oligomers ${ }^{26}$ (dimers, tetramers, pentamers and so on), known as the protomolecules. However, experimental evidence ${ }^{24,27-29}$ suggests that the protomolecules of the most common forms of eumelanin produced in laboratory have small molecular weight, supporting tetramers as the most appropriate protomolecular candidates. This state of affairs suggested to mainly focus on tetramers in the present investigation.

Once tetramers have been selected, we are still left with a large variety of possible structural models differing for the actual pattern of monomers assembling. Following the terminology introduced in a recent review on the subject, ${ }^{21}$ we refer to such a variety as the eumelanin molecular disorder. ${ }^{2,4,30}$ The tetrameric models we will consider in our work are schematically depicted in figure 2. They are just a few among all possible tetramers which have been already proved to produce the expected absorption spectrum and, therefore, receiving large attention in recent studies. ${ }^{25}$ However, they provide a trustworthy representative set as their features span the space of all molecular geometrical properties of interest for 
the present investigation, namely: cyclic and non-cyclic structure, planar and non-planar geometry. Hence, this choice for the object of investigation expectedly endows the following analysis with the necessary generality.

In addition to the molecular disorder, eumelanin structures can differ as well in the distribution of their redox states or, equivalently, in the type of constituting monomers (DHI, MQ, NQ, IQ, of figure 1): hereafter we will refer to such a feature as electronic disorder. The tetrameric models of figure 2 have, for example, the same constituting monomers (two IQs and two NQs) and a correspondingly similar electronic state. We have chosen this particular combination on the basis of a previous study by Kaxiras et al. ${ }^{31}$ for cyclic tetramers, suggesting that this particular combination should rank among the more stable ones. Both molecular and electronic disorders are considered to dictate the overall properties of eumelanin. On return, control of this disorder may permit the shaping of the material features, therefore tailoring its possible applications. ${ }^{2}$ On the other side, it is clear that the mentioned disorders are also responsible for an enormous number of possible molecular models whose complete analysis is almost unfeasible: a systematic study of thousands of possible molecular structures differing topologically and/or electronically would require, indeed, an exceedingly large computational effort.

In this work, we aim at analyzing the processes of eumelanin formation and adhesion on $\mathrm{Si}$ addressing the issue of chemical disorder. By performing a limited number of calculations on paradigmatic configurations, we derive useful information on the effects of geometrical and electronic features on molecular stability, adhesion and band line-up for efficient photoconversion. In particular, we show that the kind of constituting monomers and the planar character of the molecules represent discriminant properties in this respect.

The paper is structured as follows: after a description of the systems investigated and of the computational methods employed, we address the effects of electronic disorder of eumelanin protomolecules giving the results of the formation and adhesion energies together with the corresponding band structure. In the following section, we investigate molecular 
disorder: here different tetrameric structures are considered in a similar electronic state and formation and adhesion energies are presented. Finally, results are discussed.

\section{Computational Methods}

In this work five different protomolecular models will be considered: tetrameric models based on the work by Panzella et al., ${ }^{3}$ as well as on the cross-linked oligomers presented by Pezzella et al. ${ }^{32}$ and the cyclic porphyrin-like structure by Kaxiras et al. ${ }^{31}$ These tetramers will be hereafter referred to as Model 1, 2, .., 5, respectively. Their structure is shown in figure 2 . Thanks to the diversity of geometrical features (planarity, cyclicity and symmetries), these molecules form a meaningful and mostly representative set for the collection of all possible molecular configurations. This choice, indeed, makes the present analysis robust and accurate enough to envision general trends.

In order to study the interaction properties of eumelanin protomolecules with a substrate, we will consider adsorption on a planar slab of Si [100] having bottom dangling bonds passivated with hydrogen atoms. The surface, counting about 300 atoms, is shown in figure 3 with a tetramer adsorbed.

Density-functional theory (DFT) calculations are performed using the plane-wave Quantum Espresso package. ${ }^{33}$ The Perdew-Burke-Ernzerhof (PBE) exchange-correlation functional ${ }^{34}$ is used together with ultrasoft pseudopotentials ${ }^{35}$ and Grimme correction to include dispersive forces in the system. ${ }^{36,37}$ We used energy cutoffs of 20 Ry and 250 Ry for the wavefunction and electronic density, respectively. Structures have been relaxed until all the atomic forces were smaller than 0.001 a.u.

The calculations on the monomeric units and the complete tetrameric structures have been performed with a unit cell with the smallest linear dimension of $20 \AA$ thus leaving at least $8 \AA$ of empty space between atoms in replicated cells. Periodic boundary conditions are applied to this large supercell with the Brillouin zone sampled at the $\Gamma$ point only. In order 
to describe the effect of solvent on the formation energy, a continuum solvation model was used: the self-consistent continuum solvation model (SCCS) proposed by Andreussi, Dabo and Marzari ${ }^{38}$ and implemented in the Quantum espresso Environ tool ${ }^{33}$ has been adopted, giving an implicit description of the solvents (methanol and water in our case).

Calculations on the adhesion energies of eumelanin protomolecules on the substrate were performed using a (100) slab of Silicon atoms consisting of a $7 \times 7$ replica of the surface unit cell. In this case we sampled the Brillouin zone with a grid of $4 \times 4$ points within the Monkhorst-Pack scheme. ${ }^{39}$ Different starting molecule-surface configurations have been considered. They have been given as input to geometry optimization procedures which have been performed by fully relaxing the positions of all of the atoms in a supercell, but for the atoms of the bottom layer of the semiconductor slab which, instead, have been kept fixed to mimic the underlying bulk. The results that we will present are referred to the minimum energy configurations. Performance of the adsorbed surfaces for photovoltaic applications are eventually evaluated by calculating the system electronic structure and the density of states projected onto the molecular and surface chemical species. More specifically, the density of states is obtained projecting wavefunctions onto orthogonal atomic orbitals. HOMO and LUMO levels are consequently identified with respect to the Fermi energy and their position relatively to the surface valence and bottom bands extracted.

It is important to note that, in principle, Kohn-Sham eigenvalues of isolated (e.g., molecules) or extended (e.g., surface slabs) systems as given by the PBE functional can be affected at different extents by delocalisation errors which, in particular, lead to underestimate the band gap or the HOMO-LUMO gap. ${ }^{40,41}$ On the other hand, a correct alignment of electronic eigenvalues is crucial to provide a reliable estimate of the electronic properties of the $\mathrm{Si}$ /Eumelanin interface. Nonethless, errors to the obtained values have been shown to be small for similar systems ${ }^{42}$ and, more importantly, they are systematic; therefore, they do not affect our conclusions which are entirely based on devised trends. Notwithstanding, a comparison of band line-up of our systems computed via PBE and the hybrid BLYP 43 
functionals has been performed with ultrasoft pseudopotentials: negligible differences have been found, hence justifying the choice of PBE approach with respect to a hybrid and more computationally demanding XC functional.

In addition to the effect of the choice of the exchange-correlation functional on the bandalignment, we mention also the impact of the electric field due to the dipolar moment of the asymmetric silicon slab. Such a contribution, however, i) affects only the offset of the energy levels but not their relative difference; ii) it impacts the systems investigated here to the very same extent, not compromising the reliability of the results.

\section{Results and Discussion}

In this section we present the results of our calculations on formation energies of eumelanins and their adhesion properties on $\mathrm{Si}$. We follow the above scheme of chemical disorder of eumelanins and treat the electronic and molecular aspects separately. As it will become clear, this theoretical framework represents a convenient scheme to tackle the problem allowing to develop meaningful physical insight.

\section{Electronic disorder}

Electronic disorder relates to the distribution of redox states within the eumelanin molecules or, equivalently, to the number and type of their constituing monomers (DHI, IQ, MQ, NQ). The basic monomers are shown in figure 1: they have the same backbone, but differ in the possible presence of $=\mathrm{O}$ groups in place of $-\mathrm{OH}$. Also the $\mathrm{N}-\mathrm{H}$ fragment can be replaced by a single $\mathrm{N}$ in the monomers. The different terminations of the monomeric units naturally determine different electronic configurations of the molecule, with a consequent change in the single or double bonds among the constituting atoms. It is clear that the number of possible electronic configurations is remarkably large: e.g. for a single tetramer there are four possible oxidation monomeric forms (including the DHI monomer), implying $4^{4}=256$ 
electronic configurations. In addition, note that in some cases other tautomeric forms are also possible (see for instance model 1-a in figure 4). Performing calculations of formation and adhesion energies on all of them is impractical and we need an alternative strategy which could be neverthless of some help in understanding the effect of this disorder. In order to limit the computational cost yet offering insight on the problem, we focus on an individual tetramer. Our choice, the first molecule of figure 2, represents a good candidate for the following analysis, having no special properties (it lacks of particular symmetries and it is not cyclic) and we take it as representative of all the collection of different tetramers. For the given structural model, we consider four different electronic configurations, which are schematically depicted in figure 4. They correspond to substantially different redox states, differing in the number and location of $=\mathrm{O}$ terminations in the basic monomers (one $=\mathrm{O}$ group, two $=\mathrm{O}$ groups, four $=\mathrm{O}$ groups and $\mathrm{six}=\mathrm{O}$ groups, respectively). The formation energies of these molecules are given in table 1 in vacuum, methanol and water. The choice of methanol as solvent reflects the usual experimental conditions, being methanol the most used solvent to synthetise eumelanin. The corresponding results are then properly compared to those in water, the natural solvent by definition. Formation energies are calculated as $E_{\text {tetramer }}+3 E_{H_{2}}-\sum_{i}^{4} E_{i-t h}$ monomer , e.g. as the difference between the molecule energy and the sum of the monomeric energies (the energy of the molecular hydrogen released in the reaction is also included). The results show a clear dependence of the formation energies on the solvent actually used in the synthetization process. The presence of solvent, here taken into account via the dielectric permittivity of the medium, increases the formation energy causing the weakening of the molecular stability. Some molecular configurations considered are not formed in methanol $\left(\epsilon_{r}=32.7\right)$, while none of them is stable in water $\left(\epsilon_{r}=80.1\right)$. Moreover, the deterioration of molecular stability in solvents with high permittivity is quite general and will be noticed also in the next section for other tetrameric structures.

Another relevant information is that molecules with a high number of $=\mathrm{O}$ group terminations yield improved stability, as opposed to -OH groups which determine a higher formation 
energy. A similar conclusion had already been noticed by Kaxiras ${ }^{31}$ when presenting his own protomolecular model. However, in that case the result can be at least partially imposed by the cyclic nature of the tetramer and the fact that for steric reasons at most two nitrogen atoms can be present as $\mathrm{N}-\mathrm{H}$ groups. Looking more carefully at the results of Table 1 it seems clear that what is really important in determining the stability is the number of mono oxidized sites $(=\mathrm{O} /-\mathrm{OH})$ and that further oxidation $(=\mathrm{O} /=\mathrm{O})$ provides a weaker source of stability.

The number of $=\mathrm{O}$ terminated monomers seems to affect also the adhesion energies on a Si surface. Figure 3 shows two views of adsorbed eumelanin. The adhesion energies are calculated as the difference between the energy of the composite system and the sum of the energies of the components (non-interacting surface and molecule). We present the results in table 2 for the considered molecular structures. Stronger adhesion properties are found for molecules with more $=\mathrm{O}$ groups, highlighting the nature of chemical bonding between the molecules and the silicon atoms. Non terminated $\mathrm{O}$ atoms tend to bind to the surface, because of both charge (the oxygen atom should be more negatively charged) and overlap reasons (there is a $\mathrm{C}=\mathrm{O}$ double bond for every one of these oxygen atoms and consequently, this should favor the orbital interaction between surface and the $\pi$-type orbitals of the tetramer around that zone). The impact of $\mathrm{Si}-\mathrm{O}$ bonds in the interaction adhesion of our systems is further confirmed by the distance of the $\mathrm{O}$ atoms from the surface. This should represent a good geometrical parameter expressing the strength of the underlying adsorption process. Values of the average distance of O-atoms for our systems is given in table 3 in systems with higher number of $=\mathrm{O}$ groups, $\mathrm{O}$ atoms spatially distribute closer to the $\mathrm{Si}$ surface. The last analysis is devoted to the electronic structure properties of the eumelanin-Si systems. Figure 5 shows the Densities of States projected (PDOS) onto the eumelanin and surface chemical atoms: the green and black curves represent the density of molecular and Si states respectively, while HOMO and LUMO levels are shown as dashed lines. The continuum of states of valence and conduction bands (shaded areas) of the bulk silicon surface can easily 
be recognised as well as the energy gap. Specifically, the top (bottom) energy of valence (conduction) band of the Si surface can be accurately identified by looking at the leftmost figure where the band diagram of the pristine Si surface is given: such levels are found in correspondence of the parabolic dispersion of the energy curves. Importantly, dispersionless states in the gap denote a set of states localised at the top and bottom Si surfaces.

From the eumelanin HOMO and LUMO levels the performance of the overall system in photovoltaics can be eventually assessed. Among the considered systems, only the last one presents a proper band-alignment, with the LUMO level higher in energy with respect to silicon conduction band. In the other cases, the LUMO lies in the gap, negatively affecting the efficiency of the resulting system. Besides, a clear trend in the electronic properties can be noticed: in analogy with the previous calculations, molecules with more $=\mathrm{O}$ terminated monomers tend to show the most favourable band line-up as shown in figure 5 , hence representing good molecular candidates as photo-active layer constituents in the envisioned application. As already discussed above, these molecules, indeed, interact more strongly with the surface and this generally favours a proper band alignment. A simple tight-binding treatment of the problem of two interacting molecular systems is sufficient to explain the larger energy separation of the levels as a consequence of a stronger interaction. ${ }^{44,45}$ In our specific case, it is the interaction between the LUMO of the molecule and the higher occupied levels of the surface which is especially important. Lower will be the LUMO of the molecule, stronger will be the interaction. Increasing both the delocalization and the number of $=\mathrm{O}$ groups lowers the LUMO of the isolated molecule which, thus, will interact more strongly with the occupied levels of the Si surface. Therefore, the LUMO level will be expelled to higher energies, eventually shifting inside the conduction band.

The conclusions drawn above are quite general and independent of the geometrical parameters of the surface. In particular, when increasing the thickness of the Si slab, a reduction in the band gap should follow as a consequence of the reduced confinement effect. Consequently, the LUMO level of new molecules could fall into the conduction band. In this 
respect, our calculations represent a lower limit for the photovoltaic performances of the eumelanin/Si hybrid system. In summary, the robust conclusion is that the best molecular candidates for such an application are those with a large number of $=\mathrm{O}$ group terminations and a corresponding stronger interaction with the surface.

\section{Molecular disorder}

The other feature to be addressed when studying the performance of a eumelanin-based solar cells, is that of molecular disorder, i.e. the variety of structures obtained by unlike assembling oligomers (in our case tetramers) from the monomeric building blocks. The number of possible tetramers can be roughly estimated: assuming four possible reactive sites in each monomer where binding with other units can occur, we obtain $4 \cdot 4 \cdot(4 \cdot 3) \cdot(4 \cdot 3) \cdot(1 / 2)=$ 1152 structural models (two of the four monomers bind at only one site, the remaining

monomers at two sites). Such a large number of possibilities makes also the treatment of molecular disorder unpractical via a systematic simulative approach. Hence, we adopt a more computationally efficient strategy which allows to extract information on the effect of geometry of the protomolecular models. We identified a reduced set of members in the ensemble of all possible tetrameric configurations, which should be proper representative members of all the possibilities. They are prototypical structures representing paradigmatic characteristics, namely: planar and non-planar, as well as cyclic and non cyclic molecular structures.

In order to focus on the structural dependence of the formation and adhesion processes, we choose the molecules such that they are in a similar electronic state. Although the oxidation state may in general affect the planarity, let us remind that i) in our case, negligible modifications due to electronic state have been found in the geometry of the molecules investigated; ii) for the time being, we are interested in disclosing trends. Exploiting the conclusions of the analysis on electronic disorder, the choice of similar electronic state amounts to stating that all the molecules are given the same number of $=\mathrm{O}$ terminated groups (or, equivalently 
the same monomeric components). Specifically, in the following each molecule will have six $=\mathrm{O}$ groups (two NQ and two IQ monomers). We believe that this is the most appropriate strategy for our purpose, i.e. extracting useful information at a reasonable computational price. In addition, the similar configuration state will allow an almost fair comparison of the selected candidates. We begin calculating the formation energies of the five molecular models considered: results are shown in table 4. The effect of solvent in the formation process is again evident in the reduced values of energies, confirming the general trend previously discussed, namely: solvents with high permittivity affect the molecular stability with a consequently poorer efficiency of the eumelanin synthetisation process. The values in Table 4 reflect clearly two competing sources of stability for these molecules: (i) the delocalization within the $\pi$-system, and (ii) the tendency to minimize the steric interactions. These two effects are not independent since the type of oxidation and the number of $\mathrm{H}$ bonded to the $\mathrm{N}$ atoms are related. In models 1 and 2 delocalization is obviously the dominant source of stabilization, whereas in models 4 and 5 it is the minimization of the steric repulsions which takes the lead. Model 3 suffers from the smallest stability: this can be attributed to its geometrical configuration intrinsically leading to a stronger steric repulsion force among the out-of-plane aromatic rings.

The result which, however, reveals the most intriguing dependence on the structural parameters is represented by the adsorption on silicon. The adhesion energies of the molecules are summarized in table 5, showing the strongest adhesion for model $1 .^{3}$

The differences in adhesion energies can be traced back to the modifications of the molecular structures upon interaction with the surface atoms. In this regard, figure 6, which shows a cross-section of the different adsorbed systems, allows to recognize how the strongest interaction between molecule and surface is realized for molecules maximizing the contact surface with silicon (the number of $=\mathrm{O}$ groups closer to the surface). Molecules with an intrinsically planar character, like model 1-d and 2, are naturally favoured since a large part of their surface can be used to bind to the surface atoms. 
One remark is now in order, namely: models 1 -d and 2 reveal the strongest interaction with Si surface, being endowed with an evident planarity. However, their adhesion energies differ for a considerable quantity of about $2 \mathrm{eV}$. Such a difference can be attributed to the different internal rigidity of the molecules. The tetramer 2 has a known cyclic structure in which every constituing monomer is spatially constrained by two adjacent units. Hence, the extent to which monomers can spatially move to find the most energetically convenient configuration is limited. On the other side, the tetramer 1 has monomeric units only weakly constrained by the others and the overall structure appears more flexible. This represents a new degree of freedom for the molecule which further allows the molecule to explore the configuration space in search for more stable minima.

We conclude this section presenting the results of our calculations of the electronic structures for the systems investigated. Figure 7 shows the Density of States projected onto the molecular and surface chemical species. As it can be seen, the planar character of the eumelanin models dictates also the HOMO (LUMO)-band alignment. The planar molecules, since they can exploit all their surface and $=\mathrm{O}$ group atoms to bind to the surface, are involved in strong interaction with silicon. As already discussed, this leads to a large separation in the resulting HOMO (LUMO) levels and to a possibly improved efficiency. Specifically, models 1-d and 2 show the LUMO level above the bottom conduction band, respectively. The remaining systems, on the contrary, denote LUMO levels in the silicon energy gap, with an expected lower propensity for the application considered here. Once again, we stress here the generality of our conclusion which is independent on the geometrical features of the system.

\section{Conclusions}

In this work we investigated the formation process of eumelanin molecules and their adhesion properties on a Si substrate to address the potential of such a hybrid system for photovoltaic applications. Via DFT calculations, the formation of eumelanin protomolecular structures 
from the basic monomeric units has been assessed, disclosing the negative impact on the process played by the presence of solvent (methanol and water in our case). Adopting the chemical disorder framework, generally employed to deal with the complexity of eumelanin protomolecules, we focused our attention on tetrameric models, which are the most probable molecular candidates. Electronic and Molecular disorder have been discussed separately in order to disclose the impact of redox state and geometrical features on the properties of our applicative interest. In this respect, results demonstrate how 1) adhesion on a $\mathrm{Si}$ surface is improved by a large number of non-terminated oxygen atoms in the molecules (or equivalently, by a large number of IQ monomers); 2) intrinsically planar protomolecules generally lead to a strong interaction with the surface due to the larger contact surface. The electronic band structure of the organic/inorganic interface is affected by the mentioned molecular features as well: planar molecules with many non-terminated oxygens show the best energy levels line-up for photoconversion applications.

\section{Acknowledgement}

We acknowledge the Regione Sardegna basic research Project No. CRP78744 "Energy Applications with Porous Silicon (ENAPSi)." E.C. acknowledges support by MINECO (Spain) through Grant FIS2015-64886-C5-4-P and the Severo Ochoa Centers of Excellence Program (Grant SEV-2015-0496) as well as by Generalitat de Catalunya (2014SGR301).

\section{References}

(1) Ito, S.; Wakamatsu, K.; d'ischia, M.; Napolitano, A.; Pezzella, A. Melanins and Melanosomes; Wiley-VCH Verlag GmbH and Co. KGaA, 2011; pp 167-185.

(2) d'Ischia, M.; Napolitano, A.; Pezzella, A.; Meredith, P.; Sarna, T. Chemical and struc- 
tural diversity in eumelanins: unexplored bio-optoelectronic materials. Angew. Chem. Int. Ed. 2009, 48, 3914-3921.

(3) Panzella, L.; Gentile, G.; D’Errico, G.; Della Vecchia, N. F.; Errico, M. E.; Napolitano, A.; Carfagna, C.; d'Ischia, M. Atypical structural and $\pi$-electron features of a melanin polymer that lead to superior free-radical-scavenging properties. Angew. Chem. Int. Ed. 2013, 52, 12684-12687.

(4) Meredith, P.; Powell, B. J.; Riesz, J.; Nighswander-Rempel, S. P.; Pederson, M. R.; Moore, E. G. Towards structure-property-function relationships for eumelanin. Soft Matter 2006, 2, 37-44.

(5) Pezzella, A.; Iadonisi, A.; Valerio, S.; Panzella, L.; Napolitano, A.; Adinolfi, M.; d'Ischia, M. Disentangling eumelanin black chromophore: visible absorption changes as signatures of oxidation state-and aggregation-dependent dynamic interactions in a model water-soluble 5, 6-dihydroxyindole polymer. J. Am. Chem. Soc. 2009, 131, $15270-15275$.

(6) Mostert, A. B.; Hanson, G. R.; Sarna, T.; Gentle, I. R.; Powell, B. J.; Meredith, P. Hydration-controlled X-Band EPR spectroscopy: a tool for unravelling the complexities of the solid-state free radical in eumelanin. J. Phys. Chem. B 2013, 117, 4965-4972, PMID: 23600769.

(7) Huijser, A.; Pezzella, A.; Sundstrom, V. Functionality of epidermal melanin pigments: current knowledge on UV-dissipative mechanisms and research perspectives. Phys. Chem. Chem. Phys. 2011, 13, 9119-9127.

(8) Gauden, M.; Pezzella, A.; Panzella, L.; Neves-Petersen, M.; Skovsen, E.; Petersen, S. B.; Mullen, K.; Napolitano, A.; d'Ischia, M.; Sundstrom, V. Role of solvent, pH, and molecular size in excited-state deactivation of key eumelanin building blocks: implications for melanin pigment photostability. J. Am. Chem. Soc. 2008, 130, 17038-17043. 
(9) Bettinger, C. J.; Bruggeman, J. P.; Misra, A.; Borenstein, J. T.; Langer, R. Biocompatibility of biodegradable semiconducting melanin films for nerve tissue engineering. Biomaterials 2009, 30, 3050-3057.

(10) Pezzella, A.; Barra, M.; Musto, A.; Navarra, A.; Alfe, M.; Manini, P.; Parisi, S.; Cassinese, A.; Criscuolo, V.; d'Ischia, M. Stem cell-compatible eumelanin biointerface fabricated by chemically controlled solid state polymerization. Mater. Horiz. 2015, 2, $212-220$.

(11) Prasetyanto, E. A.; Manini, P.; Napolitano, A.; Crescenzi, O.; d'Ischia, M.; De Cola, L. Towards eumelanin zeolite hybrids: pore-size-controlled 5, 6-dihydroxyindole Polymerization. Chem. Eur. J. 2014, 20, 1597-1601.

(12) Shanmuganathan, K.; Cho, J. H.; Iyer, P.; Baranowitz, S.; Ellison, C. J. Thermooxidative stabilization of polymers using natural and synthetic melanins. Macromolecules 2011, 44, 9499-9507.

(13) McGehee, M. D. Nanostructured organic/inorganic hybrid solar cells. MRS Bull. 2009, $34,95-100$.

(14) Weickert, J.; Dunbar, R. B.; Hesse, H. C.; Wiedemann, W.; Schmidt-Mende, L. Nanostructured organic and hybrid solar cells. Adv. Mater. 2011, 23, 1810-1828.

(15) Li, G.; Zhu, R.; Yang, Y. Polymer solar cells. Nat. Photonics 2012, 6, 153-161.

(16) Mula, G.; Manca, L.; Setzu, S.; Pezzella, A. Photovoltaic properties of PSi impregnated with eumelanin. Nanoscale Res. Lett. 2012, 7, 377.

(17) Pinna, A.; Simbula, F.; Marongiu, D.; Pezzella, A.; d'Ischia, M.; Mula, G. Boosting, probing and switching-off visible light-induced photocurrents in eumelanin-porous silicon hybrids. $R S C A d v$. 2015, 5, 56704-56710. 
(18) Mayer, T.; Weiler, U.; Kelting, C.; Schlettwein, D.; Makarov, S.; Wöhrle, D.; Abdallah, O.; Kunst, M.; Jaegermann, W. Silicon/organic pigment material hybrids for photovoltaic application. Sol. Energy Mater. Sol. Cells 2007, 91, 1873-1886.

(19) Avasthi, S.; Lee, S.; Loo, Y.-L.; Sturm, J. C. Role of majority and minority carrier barriers silicon/organic hybrid heterojunction solar cells. Adv. Mater. 2011, 23, 57625766.

(20) Ambrico, M.; Ambrico, P. F.; Cardone, A.; Ligonzo, T.; Cicco, S. R.; Mundo, R. D.; Augelli, V.; Farinola, G. M. Melanin layer on silicon: an attractive structure for a possible exploitation in bio-polymer based metal-insulator-silicon devices. Adv. Mater. 2011, 23, 3332-3336.

(21) d'Ischia, M.; Napolitano, A.; Ball, V.; Chen, C.-T.; Buehler, M. J. Polydopamine and eumelanin: from structure-property relationships to a unified tailoring strategy. Acc. Chem. Res. 2014, 47, 3541-3550.

(22) Liebscher, J.; Mrówczyński, R.; Scheidt, H. A.; Filip, C.; Hadade, N. D.; Turcu, R.; Bende, A.; Beck, S. Structure of polydopamine: a never-ending story? Langmuir 2013, 29, 10539-10548.

(23) Lin, S.; Chen, C.-T.; Bdikin, I.; Ball, V.; Grácio, J.; Buehler, M. J. Tuning heterogeneous poly (dopamine) structures and mechanics: in silico covalent cross-linking and thin film nanoindentation. Soft Matter 2014, 10, 457-464.

(24) Cheng, J.; Moss, S. C.; Eisner, M.; Zschack, P. X-Ray characterization of melanins I. Pigm. Cell Res. 1994, 7, 255-262.

(25) Chen, C.-T.; Chuang, C.; Cao, J.; Ball, V.; Ruch, D.; Buehler, M. J. Excitonic effects from geometric order and disorder explain broadband optical absorption in eumelanin. Nat. Commun. 2014, 5. 
(26) Dreyer, D. R.; Miller, D. J.; Freeman, B. D.; Paul, D. R.; Bielawski, C. W. Elucidating the structure of poly (dopamine). Langmuir 2012, 28, 6428-6435.

(27) Zajac, G.; Gallas, J.; Cheng, J.; Eisner, M.; Moss, S.; Alvarado-Swaisgood, A. The fundamental unit of synthetic melanin: a verification by tunneling microscopy of X-ray scattering results. Biochim. Biophys. Acta, Gen. Subj. 1994, 1199, 271-278.

(28) Zajac, G.; Gallas, J.; Alvarado-Swaisgood, A. Tunneling microscopy verification of an xray scattering-derived molecular model of tyrosine-based melanin. J. Vac. Sci. Technol., B 1994, 12, 1512-1516.

(29) Díaz, P.; Gimeno, Y.; Carro, P.; González, S.; Schilardi, P. L.; Benítez, G.; Salvarezza, R. C.; Creus, A. H. Electrochemical self-assembly of melanin films on gold. Langmuir 2005, 21, 5924-5930.

(30) Tran, M. L.; Powell, B. J.; Meredith, P. Chemical and structural disorder in eumelanins: a possible explanation for broadband absorbance. Biophys. J. 2006, 90, 743-752.

(31) Kaxiras, E.; Tsolakidis, A.; Zonios, G.; Meng, S. Structural model of eumelanin. Phys. Rev. Lett. 2006, 97, 218102.

(32) Pezzella, A.; Panzella, L.; Natangelo, A.; Arzillo, M.; Napolitano, A.; d'Ischia, M. 5, 6-Dihydroxyindole tetramers with anomalous interunit bonding patterns by oxidative coupling of 5, 5', 6, 6'-tetrahydroxy-2, 7'-biindolyl: emerging complexities on the way toward an improved model of eumelanin buildup. J. Org. Chem. 2007, 72, 9225-9230.

(33) Giannozzi, P. et al. QUANTUM ESPRESSO: a modular and open-source software project for quantum simulations of materials. J. Phys.-Condens. Mat. 2009, 21, 395502.

(34) Perdew, J. P.; Burke, K.; Ernzerhof, M. Perdew, Burke, and Ernzerhof Reply:. Phys. Rev. Lett. 1998, 80, 891-891. 
(35) Vanderbilt, D. Soft self-consistent pseudopotentials in a generalized eigenvalue formalism. Phys. Rev. B 1990, 41, 7892-7895.

(36) Grimme, S. Semiempirical GGA-type density functional constructed with a long-range dispersion correction. J. Comput. Chem. 2006, 27, 1787-1799.

(37) Barone, V.; Casarin, M.; Forrer, D.; Pavone, M.; Sambi, M.; Vittadini, A. Role and effective treatment of dispersive forces in materials: polyethylene and graphite crystals as test cases. J. Comput. Chem. 2009, 30, 934-939.

(38) Andreussi, O.; Dabo, I.; Marzari, N. Revised self-consistent continuum solvation in electronic-structure calculations. J. Chem. Phys. 2012, 136, 064102.

(39) Monkhorst, H. J.; Pack, J. D. Special points for Brillouin-zone integrations. Phys. Rev. B 1976, 13, 5188 .

(40) Cohen, A. J.; Mori-Sánchez, P.; Yang, W. Insights into current limitations of density functional theory. Science 2008, 321, 792-794.

(41) Mori-Sánchez, P.; Cohen, A. J.; Yang, W. Localization and delocalization errors in density functional theory and implications for band-gap prediction. Phys. Rev. Lett. 2008, 100, 146401.

(42) Mattioli, G.; Filippone, F.; Alippi, P.; Giannozzi, P.; Bonapasta, A. A. A hybrid zinc phthalocyanine/zinc oxide system for photovoltaic devices: a DFT and TDDFPT theoretical investigation. J. Mater. Chem. 2012, 22, 440-446.

(43) Becke, A. D. Density-functional thermochemistry. III. The role of exact exchange. $J$. Chem. Phys. 1993, 98, 5648-5652.

(44) Ashcroft, N.; Mermin, N. Solid State Physics; Cengage Learning, 2011.

(45) Rössler, U. Solid state theory: an introduction; Springer Science \& Business Media, 2009 . 
Table 1: Formation enegies $[\mathrm{eV}]$ of tetrameric Model 1 in the four electronic configurations of figure 4. Values in vacuum, methanol and water are given.

\begin{tabular}{rrrr}
\hline Formation energy $[\mathrm{eV}]$ & Vacuum & Methanol & Water \\
\hline Model 1-a $($ one $=\mathrm{O})$ & -0.56 & -0.12 & 0.67 \\
Model 1-b (two $=\mathrm{O})$ & -0.26 & 0.35 & 0.74 \\
Model 1-c $($ four $=\mathrm{O})$ & -1.20 & -0.07 & 0.92 \\
Model 1-d $($ six $=\mathrm{O})$ & -1.46 & -0.20 & 0.91 \\
\hline
\end{tabular}

Table 2: Adhesion energies of tetrameric Model 1 in the four electronic configurations of figure 4 .

\begin{tabular}{rr}
\hline Molecule & Adhesion Energy $[\mathrm{eV}]$ \\
\hline Model 1-a $($ one $=\mathrm{O})$ & -5.37 \\
Model 1-b $($ two $=\mathrm{O})$ & -6.73 \\
Model 1-c $($ four $=\mathrm{O})$ & -7.85 \\
Model 1-d $($ six $=\mathrm{O})$ & -10.99 \\
\hline
\end{tabular}

Table 3: Average distance of Oxygen atoms from the silicon surface of tetrameric Model 1 in the four electronic configurations of figure 4 .

\begin{tabular}{rr}
\hline Molecule & Average O-distance $[\AA]$ \\
\hline Model 1-a $($ one $=\mathrm{O})$ & 2.40 \\
Model 1-b $($ two $=\mathrm{O})$ & 2.39 \\
Model 1-c $($ four $=\mathrm{O})$ & 2.20 \\
Model 1-d $($ six $=\mathrm{O})$ & 1.96 \\
\hline
\end{tabular}


Table 4: Formation energies of tetrameric Models of figure 2.

\begin{tabular}{rrrr}
\hline Molecule & Form. Energy-vacuum $[\mathrm{eV}]$ & Methanol & Water \\
\hline Model 1 & -1.46 & -0.20 & 0.90 \\
Model 2 & -1.64 & -0.22 & 0.73 \\
Model 3 & -0.34 & 0.59 & 0.97 \\
Model 4 & -1.18 & -0.11 & 0.39 \\
Model 5 & -1.94 & -0.43 & 0.25 \\
\hline
\end{tabular}

Table 5: Adhesion energies of tetrameric Models of figure 2.

\begin{tabular}{rr}
\hline Molecule & Adhesion Energy. $[\mathrm{eV}]$ \\
\hline Model 1 & -11.02 \\
Model 2 & -8.93 \\
Model 3 & -7.22 \\
Model 4 & -6.93 \\
Model 5 & -8.63 \\
\hline
\end{tabular}

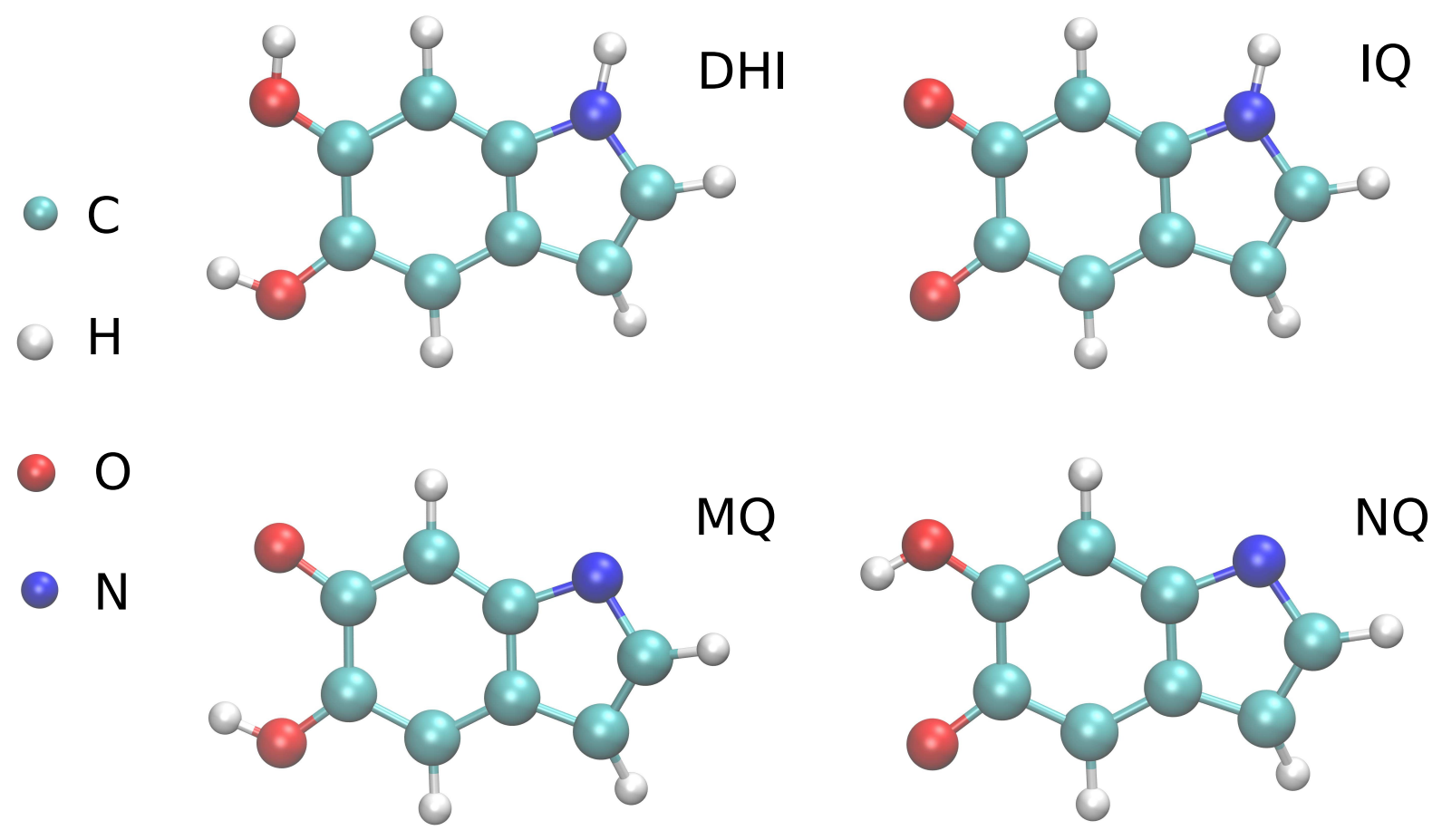

Figure 1: Monomeric species involved in eumelanins that are considered in the present work: 5,6-dihydroxy-indole (DHI) and its three redox forms (indolequinone (IQ), quinone-methide (MQ) and quinone-imine (NQ)). 


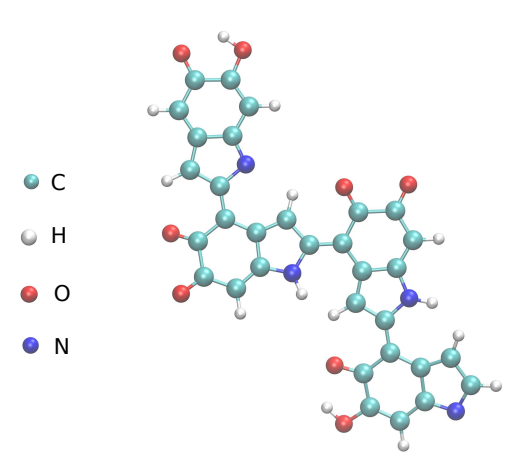

Model 1-d

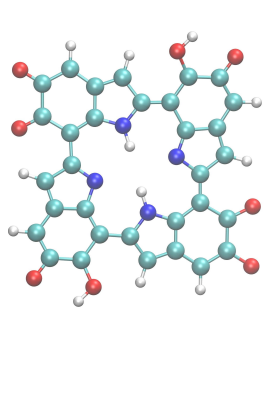

Model 2
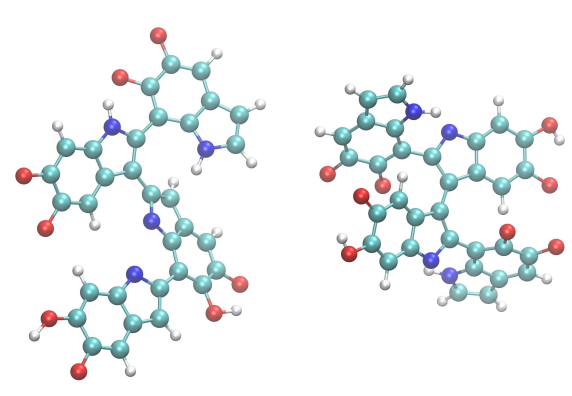

Model 3

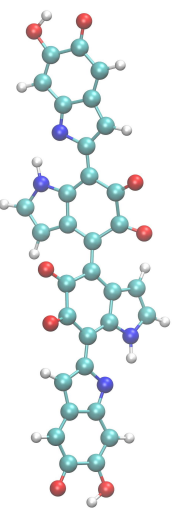

Model 4

Model 5

Figure 2: Eumelanin tetramers considered in this work. The corresponding precursor forms of the same tetrameric models (not shown here) have the same structure with all reactive sites terminated with $\mathrm{OH}$ groups.

a)

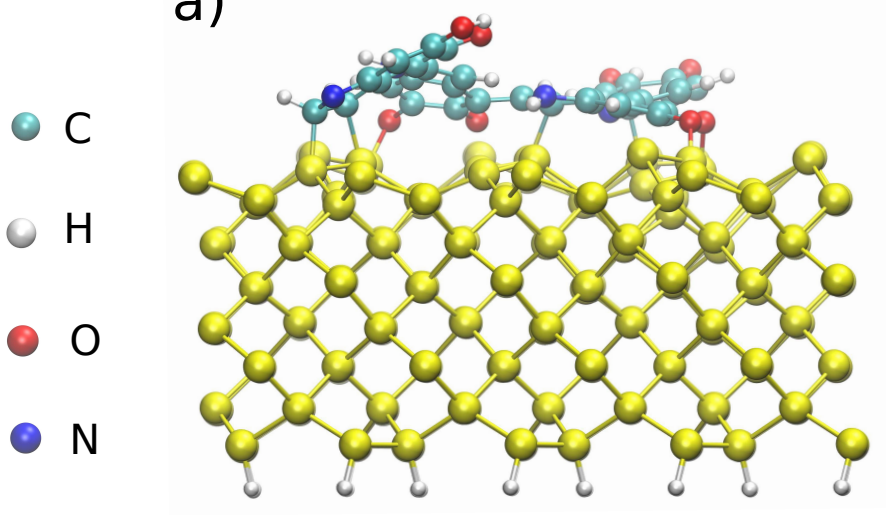

O Si b)

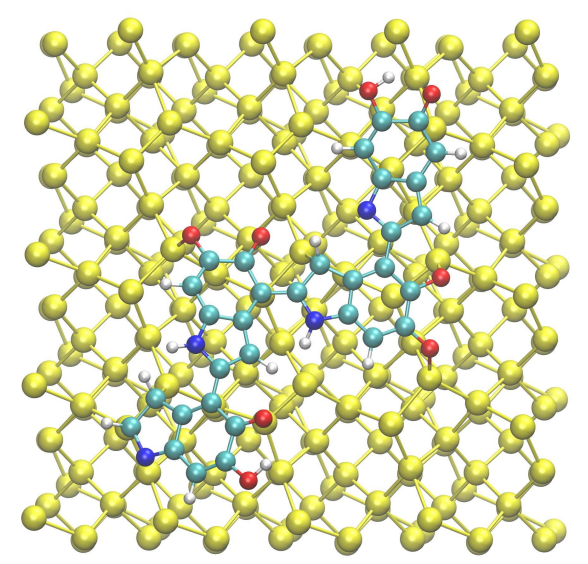

Figure 3: Adsorption of tetrameric model 1-d by Panzella ${ }^{3}$ on a Si surface. The full system with the slab of the surface supercell used in the calculations is displayed: (a) side view, (b) top view. The bottom atoms are Hydrogen-passivated. 


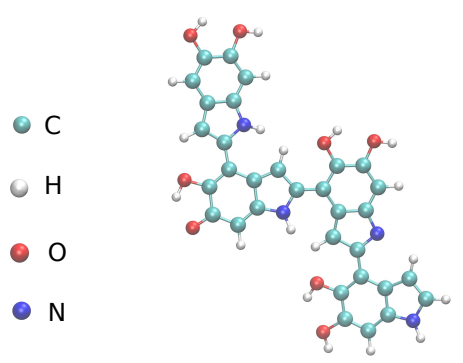

Model 1-a

( $1=0$ group $)$

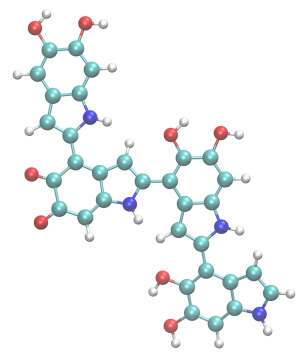

Model 1-b

( $2=0$ groups $)$

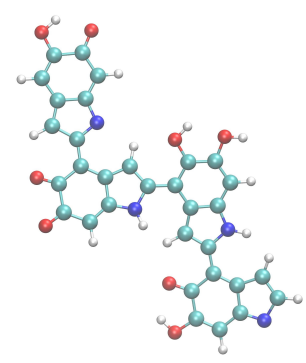

Model 1-c

$(4=0$ groups $)$

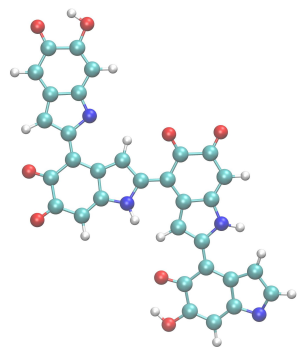

Model 1-d

$(6=0$ groups $)$

Figure 4: Tetrameric model 1 in four different electronic configurations. The redox states showed present one, two, four and six $=\mathrm{O}$ groups, respectively. They are referred in the text as Model 1-a, 1-b, 1-c and 1-d.
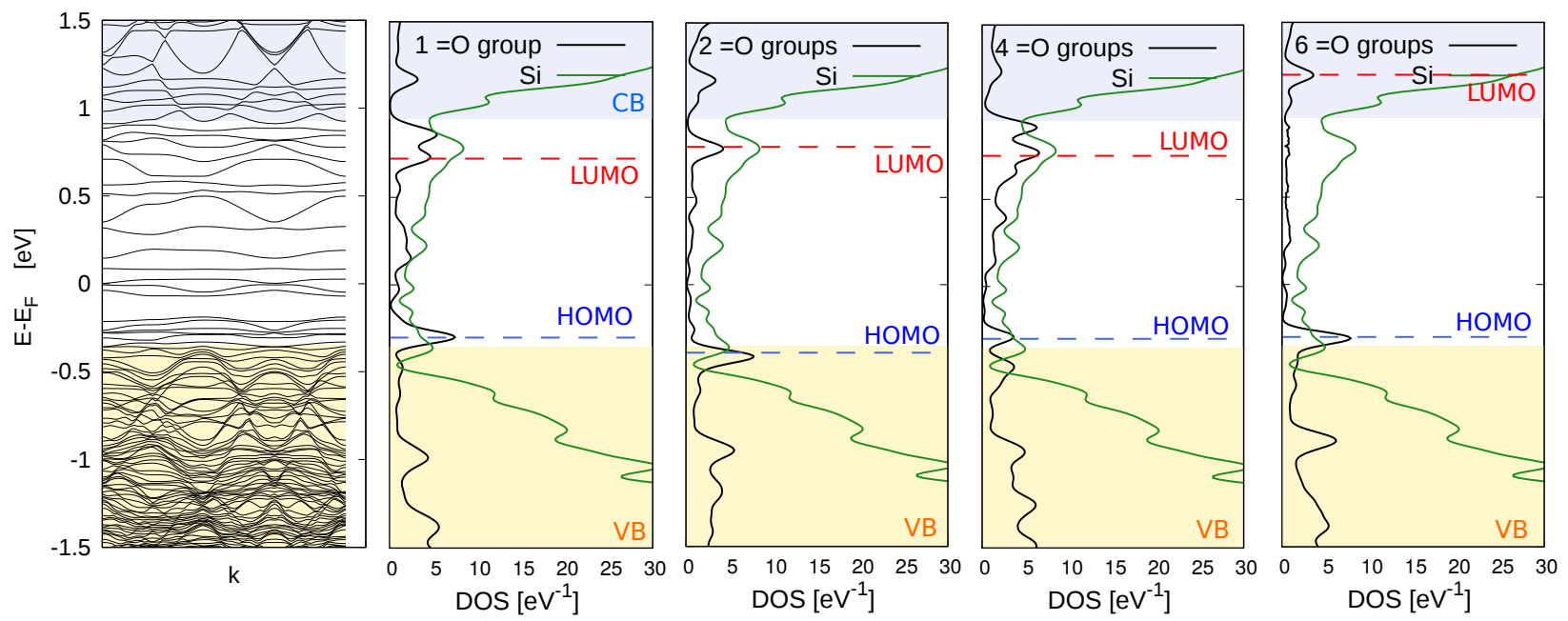

Figure 5: Density of states (DOS) projected onto the molecular and surface chemical species for the different electronic configurations. Black and green curves represent the density of molecular and silicon states, respectively; HOMO and LUMO levels are shown via dashed lines in the figure, while shaded areas identify conduction (in gray) and valence bands (orange). In the left-most figure the band diagram of the pristine silicon surface is shown: valence and conduction bands correspond to parabolic dispersion curves (shaded areas). On the contrary, dispersionless states inside the gap denote electronic states localised on the top and bottom silicon surfaces. 

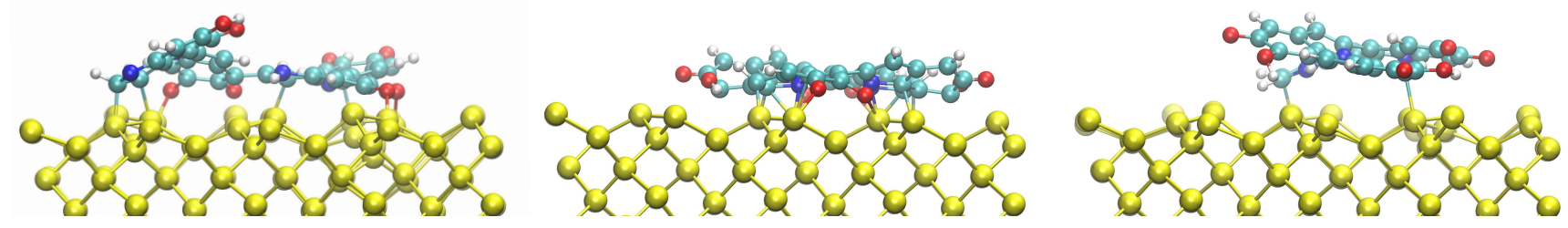

Model 1

Model 2

Model 3
- C
o $\mathrm{H}$
- $\mathrm{O}$
- $\mathrm{N}$
- $\mathrm{Si}$

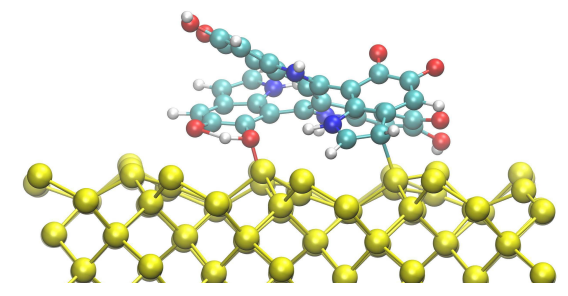

Model 4

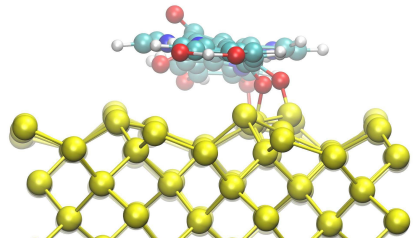

Model 5

Figure 6: Side views of the relaxed silicon surfaces adsorbing the tetrameric models considered in this section. 

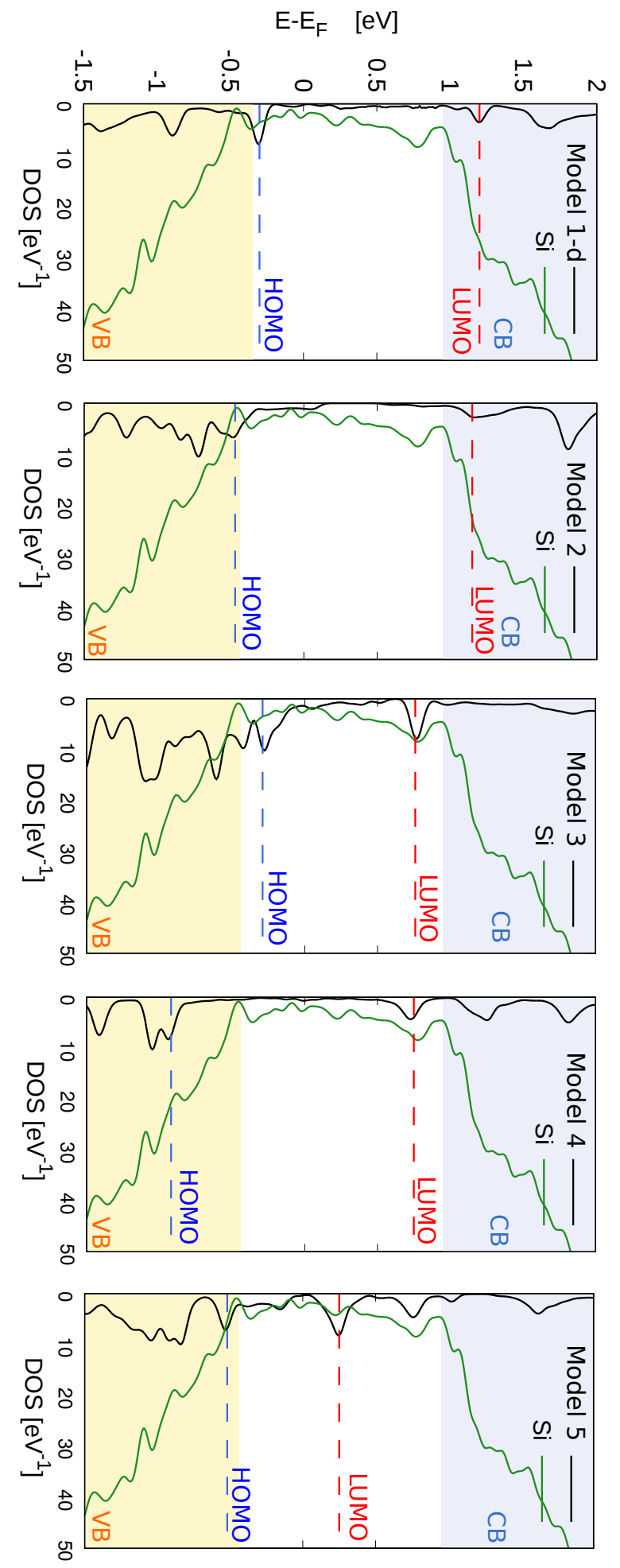

Figure 7: Density of states projected onto the molecular and surface chemical species for the different structural models. Black and green curves represent the density of molecular and silicon states, respectively; HOMO and LUMO levels are shown via dashed lines in the figure, while shaded areas identify conduction (in gray) and valence bands (orange). Top valence (bottom conduction) energies are found by direct comparison with the corresponding band diagrams (not shown here). 


\section{Graphical TOC Entry}

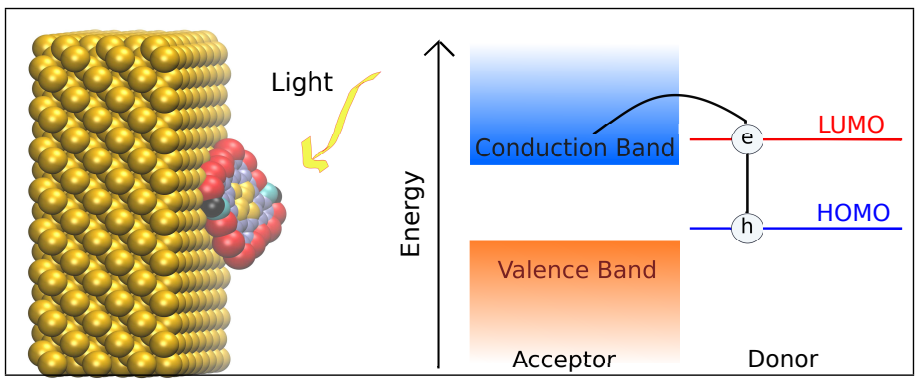

